\title{
Relation Between The Roughness, Linear Entropy and Visibility of A Quantum State, The Jaynes- Cummings Model.
}

Adélcio Carlos de Oliveira ( $\square$ adelcio@ufsj.edu.br)

Universidade Federal de São João del-Rei: Universidade Federal de Sao Joao del-Rei https://orcid.org/0000-0001-5190-6521

Mauricio Reis

Universidade Federal de São João del-Rei: Universidade Federal de Sao Joao del-Rei

\section{Research Article}

Keywords: Entropy, Entanglement, Wigner Functions

Posted Date: July 12th, 2021

DOl: https://doi.org/10.21203/rs.3.rs-581828/v1

License: (c) (i) This work is licensed under a Creative Commons Attribution 4.0 International License.

Read Full License

Version of Record: A version of this preprint was published at Journal of Computational Electronics on August 19th, 2021. See the published version at https://doi.org/10.1007/s10825-021-01761-0. 


\title{
Relation between the Roughness, Linear Entropy and Visibility of a quantum state, the Jaynes-Cummings Model.
}

\section{Mauricio Reis and Adélcio C. Oliveira}

Received: date / Accepted: date

\begin{abstract}
In this work, an analysis of the Jaynes-Cummings Model is conducted in the parameter spaces, composed of Roughness, Concurrence/Linear Entropy and Visibility. The analysis was carried out without including the effects of the environment and with the inclusion of a dispersive environment. As Roughness measures the state's degree of non-classicality, its inclusion in the analysis allows to identify points in the dynamics that are not usually perceived by traditional analysis. It is observed that the parameter space is almost completely occupied when the dispersive term is small, and is concentrated in the region of less roughness and less purity as the dispersive coefficient is increased.
\end{abstract}

Keywords Entropy, Entanglement, Wigner Functions

\section{Introduction}

Ever since its first formulation, Quantum Mechanics has amazed scientists by its counter intuitive results and predictions [1,2]. Starting with the transistor and up to nowadays quantum information and computation, technology development based on this results and predictions lead us to marvelous devices and ways of processing information, such as quantum cryptography [3], generation of random numbers [4], quantum teleportation [6], and quantum computation [5]. At the heart of such applications are quantum effects which would never be obtained by classical methods or devices, these quantum features used to obtain some advantage in a specific task are now recognised as quantum resources [7]. With the increasing demand for new ways to achieve quantum computation, it comes to our attention how can we find useful states and dynamics capable of generating special quantum effects. To help in achieving this goal, one can use quantifiers or indicators of "quantumness", i.e., how

Departamento de Estatística, Física e Matemática, Universidade Federal de São João Del Rei, C.P. 131, Ouro Branco, MG, 36420 000, Brazil 
physical properties which are strictly quantum features can be sought in a given state and/or dynamics. For this purpose, many quantifiers were proposed for different contexts, for some examples see [8] and references there in. Considering non-locality effects of quantum mechanics, a property well studied, explored and characterized is the quantum entanglement between two or more sub-systems. This property has once puzzled Einstein and lead to an important debate between him and Bohr regarding the physical meaning and interpretation of the early developed quantum theory [9]. Quantum entanglement were later carefully studied by Bell which shown, by using inequalities, how quantum mechanics defies our classical interpretation of probabilities and mean values of correlated quantities in this case [10]. Nowadays, we already have quantum devices exploring the entanglement of a 2-part system to deploy a new cryptography protocol immune to eavesdropping [3] and another outstanding application of this non-locality is quantum teleportation [6].

Quantum effects, however, are not limited to the non-locality of a multipart system, they can also be found locally, in systems described by a single part. Perhaps one of the most popular of this systems is the Schrödinger cat state, which is a quantum correlated superposition of two coherent states. Although the original purpose of Schrödinger thought experiment was to illustrate a paradox of quantum superpositions, actually there are many experimental realization of these "strange" estate, some examples are the Schrödinger cat states using atom arrays[11,13] and photons [12]. Even when looked locally, statistical measures of physical quantities, like position and momentum, shows non-classical results [14], and the classical limit is achieved with a combination of factors, the action of an environment [14], on the observable choice [15] and on how used measurement process [16] is performed.

For both local and global aspects of quantum mechanics, there are ways to quantify how much the properties of a system have intrinsic quantum or classical features. For measuring entanglement between the parts of a system, there are several functions like Concurrence [17] and other entropic measures $[19,20]$. For local aspects, we can look into phase space representations given by Wigner and Husimi functions and search for features not found in its corresponding statistical counter part. A quantum phase transition can be identified in term of Wigner function, and quantified by linear entropy [21], and there is also at least one entropic measure based on Wigner function [22]. Historically, it was done by the Negativity of Wigner functions [23], and more recently a measure of quantumness was proposed by the Roughness [24]. Another potential function is the Wigner-Yanase skew information (WYSI) measure [25]. For the purpose of the current work, Roughness were chosen as quantumness indicator since, unlike Negativity and WYSI, its values are limited to the $[0,1]$ interval and it is able to detect quantum features not detected by Negativity. Furthermore, Roughness can be obtained for any state as a summation of analytical terms.

The objective of this work is to study the relationship between local quantum features and correlations between the parts of a 2-qubit system. The used dynamics is governed by the Jaynes-Cummings model [26], which represents 
two interacting quantum systems: a two-level atom and a single mode of the radiation field, with a complex but analytically solvable dynamics $[27,28]$. To start working with it, let us first make a brief review of the used quantifiers.

\section{Classicality Quantifiers}

\subsection{Linear Entropy}

For a bipartite system represented by a global density matrix $\rho$, the reduced density matrix operators $\rho_{A}=\operatorname{Tr}_{F} \rho$ and $\rho_{F}=\operatorname{Tr}_{A} \rho$ have the information contained in each subsystem separately, accessible through local measurements in each part. In the special case where the quantum information of a pure global state can be completely reconstructed by the information contained in the corresponding reduced density matrix operators, the sum of the parts contains the whole and there is no quantum correlation between them. When it happens, $\rho_{A}^{2}=\rho_{A}, \rho_{F}^{2}=\rho_{F}$ and:

$$
\operatorname{Tr}\left\{\rho_{A}^{2}\right\}=\operatorname{Tr}\left\{\rho_{F}^{2}\right\}=1
$$

For all other cases, however, the sum of the parts is not equal to the whole, and quantum correlations holds part of the information. A simple way to measure these quantum correlations between parts of a bipartite system is, then, to check how much these reduced density matrix operators have departed from being true projectors. It can be done by the Linear Entropy:

$$
\delta=1-\operatorname{Tr}\left\{\rho_{A}^{2}\right\},
$$

so, for a pure $\rho$ with no quantum correlation between its parts, $\delta=0$. If, however, there is quantum correlation between subsystems, the partial trace operation in (2) will result in a non-pure $\rho_{A}$ and $0<\delta<1$.

\subsection{Visibility, Concurrence and Predictability}

Concurrence is a measure of quantum entanglement. As proposed by Wooters [17], to obtain the Concurrence of a two-qubit system represented by a density state $\rho$, we initially define a flipped form $\tilde{\rho}=\left(\sigma_{y} \otimes \sigma_{y}\right) \rho^{*}\left(\sigma_{y} \otimes \sigma_{y}\right)$, where $\rho^{*}$ is the complex conjugate of $\rho$. The concurrence can, then, be obtained by:

$$
C(\rho)=\max \left\{0, \lambda_{1}-\lambda_{2}-\lambda_{3}-\lambda_{4}\right\},
$$

where $\left\{\lambda_{i}\right\}_{i=1}^{4}$ are the eigenvalues of the matrix $R \equiv \sqrt{\sqrt{\rho} \tilde{\rho} \sqrt{\rho}}$, written in decreasing order. The Concurrence of a globally pure state $\rho$ can be calculated from its linear entropy by the following relation:

$$
C(\rho)=\sqrt{2 \delta(\rho)} .
$$

This relationship can also work as a generalization of Concurrence for higher dimensional systems [18]. 
The Visibility of a two level system can be determined by

$$
V=2\left|\left\langle\sigma_{+}\right\rangle\right| \text {. }
$$

For a global pure state, the Visibility, Concurrence and Predictability obeys the relation

$$
V^{2}+C^{2}+P^{2}=1,
$$

where $P=\left|\left\langle\sigma_{z}\right\rangle\right|$, see [29]. The Concurrence $C$ can be obtained by the relation (6). Recently, this relation was experimentally investigated and there is an evidence of its validity in a bipartite quantum system with superconducting qubits in the IBM Q quantum computer [30].

\subsection{Roughness}

Roughness was proposed by Lemos et. al. [24] as indicator of quantum properties in a given system which departs from its classical analogue. It uses the phase space representations of Wigner and Husimi functions, being defined for a given density state matrix $\rho$ by:

$$
R(\rho)=\sqrt{2 \pi \int_{\Re^{2}} d q d p\left|W_{\rho}(q, p)-Q_{\rho}(q, p)\right|^{2}},
$$

where $W_{\rho}(q, p)$ and $H_{\rho}(q, p)$ are the Wigner and Husimi representations of $\rho$ and the integral is done over the complete phase space. As stated by equation (7), Roughness measures how the two representations, Wigner and Husimi, differ from each other, summing up the absolute value of the difference. The resulting measure is bounded to the interval $[0,1]$, with $R=0$ meaning a completely classical state and $R=1$ corresponding to the maximum of "quantumness" achievable for a given state. To illustrate this, we can state some examples:

- the Roughness of Fock states increases with their eigenvalues $n$, with $R \rightarrow 1$ as $n \rightarrow \infty$, and the fundamental state corresponding to $n=0$ having the lesser value of Roughness from this set of states, corresponding to $R_{0}=1 / \sqrt{6}$. This value for the $n=0$ Fock state is also the value of Roughness for any coherent state, as should be expected;

- the Roughness of Squeezed States increase with the absolute values of the squeezing parameter;

- the Roughness of superposed pure coherent states of the form $|\psi\rangle=\left[\left|q_{0}\right\rangle \pm\right.$ $\left.\left|-q_{0}\right\rangle\right] / \sqrt{2}$ (Schrödinger's Cat States) increases with $q_{0}$, having the odd superposition greater Roughness if compared with the even one, but both converging to a maximum value of $R=\sqrt{7 / 12}$ as $q_{0} \rightarrow \infty$;

- the Roughness of thermal states goes to zero as their corresponding temperature increases;

- Thermal and diagonal states have different Roughness. 
Following [24], we can calculate the Roughness of a general state of the form

$$
\rho=\sum_{n m} A_{n m}|n\rangle\langle m|
$$

by using:

$R^{2}(\rho)=\sum_{n m, n^{\prime} m^{\prime}} A_{n m}^{*} A_{n^{\prime} m^{\prime}}\left[R_{\Pi_{m n} \Pi_{m^{\prime} n}}^{2}+R_{\Psi_{m n} \Psi_{m^{\prime} n}}^{2}-\left(R_{\Pi_{m n} \Psi_{m^{\prime} n}}^{2}+R_{\Psi_{m n} \Pi_{m^{\prime} n}}^{2}\right)\right]$

where the various terms within the brackets are results of the evaluation of integrals. According to the Appendix $\mathrm{C}$ of the referenced article, we have:

$$
\begin{aligned}
R_{\Pi_{m n} \Pi_{m^{\prime} n^{\prime}}}^{2} & =\delta_{n n^{\prime}} \delta_{m m^{\prime}} \\
R_{\Psi_{m n} \Psi_{m^{\prime} n}}^{2} & =\frac{\delta_{n-m, n^{\prime}-m^{\prime}}}{\sqrt{n ! m ! n^{\prime} ! m^{\prime} !}}\left(\frac{1}{2}\right)^{1+v} v ! \\
R_{\Pi_{m n} \Psi_{m^{\prime} n^{\prime}}}^{2} & =\frac{2 \delta_{n-m, n^{\prime}-m^{\prime}}}{3}(-1)^{Y} \sqrt{\frac{Y !}{X ! Y ! Y^{\prime} !}} 2^{X-Y}\left(\frac{1}{3}\right)^{u}{ }_{2} F_{1}\left[\begin{array}{c}
-Y, u+1 \\
X-Y+1
\end{array} ; 4 / 3\right] \\
R_{\Pi_{m n} \Psi_{m^{\prime} n^{\prime}}}^{2} & =\frac{2 \delta_{n-m, n^{\prime}-m^{\prime}}}{3}(-1)^{Y^{\prime}} \sqrt{\frac{Y^{\prime} !}{X ! Y ! X^{\prime} !}} 2^{X^{\prime}-Y^{\prime}}\left(\frac{1}{3}\right)^{u^{\prime}}{ }_{2} F_{1}\left[\begin{array}{c}
-Y^{\prime}, u^{\prime}+1 \\
X^{\prime}-Y^{\prime}+1
\end{array} ; 4 / 3\right]
\end{aligned}
$$

where $X$ and $Y$ stands for $\max (n, m)$ and $\min (n, m)$, with the corresponding relations for the primed indexes, $u=\left(X-Y+X^{\prime}+Y^{\prime}\right) / 2, v=(n+m+$ $\left.n^{\prime}+m^{\prime}\right) / 2$ and ${ }_{2} F_{1}$ is the Generalized Hypergeometric Function using the bracketed arguments.

\subsubsection{Physical insight of Roughness}

As Wigner function has all information about the state and Husimi function can approximately be obtained from classical trajectories [31,32], then Roughness can be interpreted as a distance of the quantum state from the most classical positive quantum distribution. In fact, Roughness is close related to Nearest Hilbert-Schmidt distance [33], which is defined as the minimum distance from a coherent state, the difference is that a Gaussian state is not usually the classical distribution [34-38].

\subsubsection{Roughness of a bipartite entangled system}

The Roughness, as a measure of non-classicality of a state, is related to the concurrence of a qubit, as has already been observed in the subspace generated by 3 of the Bell states, as shown by [39]. In that work, a generalization 
of Roughness to measure local quantum features in a system of two qubits represented by a density matrix $\rho$ is defined as:

$$
R_{+}(\rho)=\frac{\sqrt{R^{2}\left(\rho_{A}\right)+R^{2}\left(\rho_{B}\right)}}{\sqrt{2}},
$$

where $R^{2}\left(\rho_{A, B}\right)$ stands for the squared Roughness of the reduced density matrix obtained by tracing over the other part.

\section{The Jaynes-Cummings model}

E.T. Jaynes and F. W. Cummings proposed a model (JC-model), to investigate the interactions of a two level atom and the electromagnetic field using a semi-classical approach [26]. Considering a single, resonating mode of the electromagnetic field, the corresponding Hamiltonian is obtained considering the sum of $\mathbf{H}_{\mathbf{0}}$, representing the number of excitation in the atom and the field, with $\mathbf{H}_{\mathbf{I}}$, an interaction between the atom and the field with coupling parameter $g$ :

$$
\mathbf{H}_{\mathbf{J C}}=\frac{1}{2} \hbar \omega \sigma_{z}+\hbar \omega \mathbf{a}^{\dagger} \mathbf{a}+\hbar g\left(\sigma_{+} \mathbf{a}+\sigma_{-} \mathbf{a}^{\dagger}\right),
$$

where $\mathbf{a}^{\dagger}$ and $\mathbf{a}$ are creation and annihilation bosonic operators for the field and $\sigma_{z}, \sigma_{ \pm}$are spin- $1 / 2$ operators acting on the atom subsystem. The ground, $|0\rangle$, and excited, $|1\rangle$, states of the atom are eigenstates of $\sigma_{z}$. Using these two states to form a basis, $\sigma_{z}=|1\rangle\langle 1|-| 0\rangle\left\langle 0\left|, \sigma_{+}=\right| 1\right\rangle\langle 0|$ and $\sigma_{-}=|0\rangle\langle 1|$. The Hilbert space used to describe the quantum dynamics of this system is a direct product of the atom and field Hilbert spaces, $\mathcal{H}=\mathcal{H}_{\mathcal{A}} \otimes \mathcal{H}_{\mathcal{F}}$, where $\mathcal{H}_{\mathcal{A}}$ can be generated by the $\{|0\rangle,|1\rangle\}$ basis and $\mathcal{H}_{\mathcal{F}}$ can be generated by $\{|n\rangle\}_{n=0}^{\infty}$, the Fock states basis. This way, any initial state therein can be written as:

$$
|\Psi(0)\rangle=\left(C_{e}|1\rangle+C_{g}|0\rangle\right) \otimes\left(\sum_{n=0}^{\infty} C_{n}|n\rangle\right) .
$$

Since $\mathbf{H}_{\mathbf{J C}}$ commutes with $\mathbf{H}_{\mathbf{0}}$, block diagonalization can be used to obtain eigenstates with definite number of excitations and give the time evolution of the general initial states above:

$$
\begin{array}{r}
|\Psi(t)\rangle=\sum_{n=0}^{\infty}\left\{\left(C_{e} C_{n} \cos (g t \sqrt{n+1})-i C_{g} C_{n+1} \sin (g t \sqrt{n+1})\right)|1\rangle+\right. \\
\left.\left(-i C_{e} C_{n-1} \sin (g t \sqrt{n})+C_{g} C_{n} \cos (g t \sqrt{n})\right)|0\rangle\right\} \otimes|n\rangle .
\end{array}
$$

Although analytically solvable, the Jaynes-Cummings model presents rich dynamics. A deeper discussion about the model application and its implementation can be found on [40]. 


\section{Dynamics in the Parameter space}

Given the various quantifiers, a graphical analysis can be used for a better understanding of the quantum effects. As long as we are dealing with a bipartite system, we ought to correlate the local and global quantum features introduced so far: Roughness, Visibility, Concurrence and Linear Entropy.

The time evolution will be given by equation (15) with $g=\omega / 2$ and in all cases we will be considering an initial pure 2-qubits state of the form:

$$
|\psi(0)\rangle=(\alpha|1\rangle+\beta|0\rangle) \otimes\left(c_{1}|1\rangle+c_{0}|0\rangle\right) .
$$

Since this is a direct product of the atom and the field, it can be thought of as representing a condition where the available global information were formerly known from atom and field separate preparations. Thus, in this case, the initial linear entropy of the system is initially null.

The time evolution of the atom subsystem $\left(\rho_{A}\right)=\operatorname{Tr}_{F} \rho_{A}$ will always has the form:

$$
\rho_{A}(t)=\sum_{k=0}^{\infty}\left(\begin{array}{cc}
\left|B_{k}(t)\right|^{2} & B_{k}(t) A_{k}^{*}(t) \\
B_{k}^{*}(t) A_{k}(t) & \left|A_{k}(t)\right|^{2}
\end{array}\right)
$$

where

$$
\begin{aligned}
& A_{k}(t)=\alpha c_{k} \cos (g t \sqrt{k+1})-i \beta c_{k+1} \sin (g t \sqrt{k+1}), \\
& B_{k}(t)=-i \alpha c_{k-1} \sin (g t \sqrt{k})+\beta c_{k} \cos (g t \sqrt{k}) .
\end{aligned}
$$

As the atom interacts with the field, however, different dynamics for the global system will emerge according to how many excitations are avaiable to the global system.

\subsection{One Excitation Subspace}

For the first case study, consider the initial state obtained by setting $\alpha=0$, $\beta=1$ and $c_{1}=c_{2}=1 / \sqrt{2}$ in (18):

$$
\left|\psi_{1}\right\rangle=|0\rangle \otimes\left(\frac{1}{\sqrt{2}}|0\rangle+\frac{1}{\sqrt{2}}|1\rangle\right) .
$$

Figure 1 shows the Wigner representation of $\left|\psi_{1}\right\rangle$ as a color map.

In this case, the dynamics is restricted to the subspace with less than one excitation, the field states with $n>1$ will never get populated and the time evolution of field subsystem can be described by a $2 \times 2$ square matrix:

$$
\rho_{F}(t)=\left(\begin{array}{cc}
\left|A_{0}(t)\right|^{2}+\left|B_{0}(t)\right|^{2} & B_{0}(t) B_{1}^{*}(t)+A_{0}(t) A_{1}^{*}(t) \\
B_{1}(t) B_{0}^{*}(t)+A_{1}(t) A_{0}^{*}(t) & \left|A_{1}(t)\right|^{2}+\left|B_{1}(t)\right|^{2}
\end{array}\right) .
$$

Also, since the global state is a 2-qubit for the entire dynamics, we shall use the Concurrence as the measure of quantum entanglement. This dynamics is simply periodic with a single factor of $\omega$, then the Concurrence, Roughness 

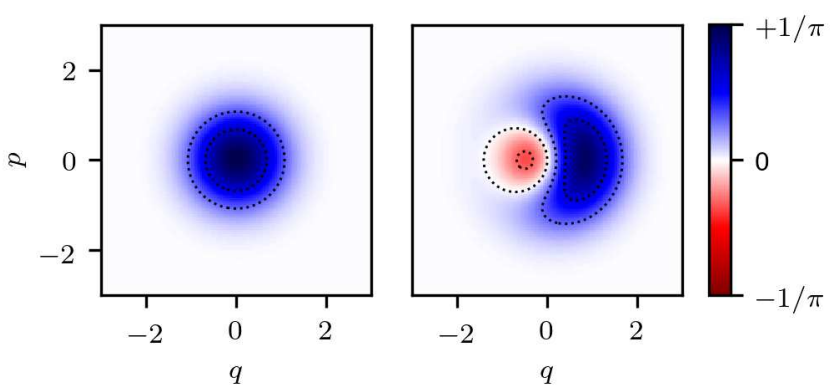

Fig. 1 The atom (left) and field (right) Wigner Functions for $\left|p s i_{1}\right\rangle$ in equation (21).

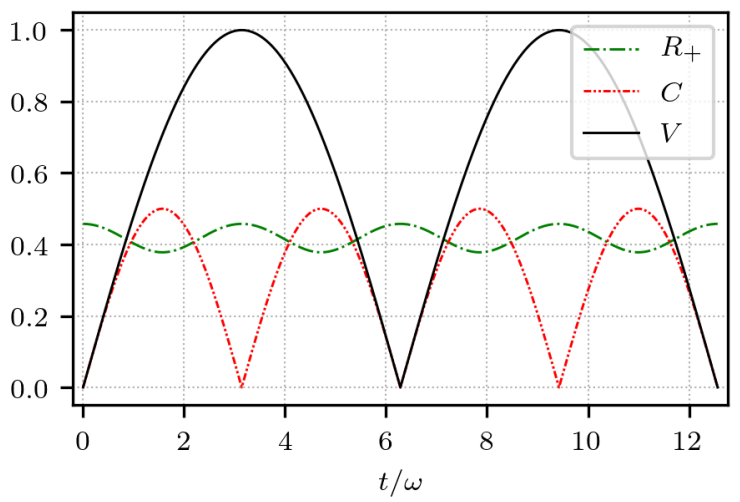

Fig. 2 Time evolution of Concurrence, $C$, combined Roughness, $R_{+}$of equation (14), and Visibility, $V$, for the initial state $\left|\psi_{1}\right\rangle$. given in equation (21).

and Visibility are also periodic, as shown in figure 2. The relation between these quantities will also be simply periodic, as shown in figure 3, generating a simple curve without crossings for each pair. The maximum of Concurrence occurs when $t=\frac{1}{2}(n+1) \pi \omega^{-1}$, for $n \in \mathbb{Z}$, times at which $R_{+}$is minimum, and $R_{A}=R_{F}$. Figure 4 shows the Wigner functions of each sub-system for one of this times.

In figure 2 one can also see the periodic zeroes of Concurrence, corresponding to the states: $\rho_{A}=|1\rangle\langle 1|$ or $\rho_{A}=|0\rangle\langle 0|$, occurring whenever $t=\frac{1}{2} n \pi \omega^{-1}$, for $n \in \mathbb{Z}$.

\subsection{Two Excitation Subspace}

We consider now a initial state of the form:

$$
\left|\psi_{2}\right\rangle=\left(\frac{1}{\sqrt{2}}|0\rangle+\frac{1}{\sqrt{2}}|1\rangle\right) \otimes\left(\frac{1}{\sqrt{2}}|0\rangle+\frac{1}{\sqrt{2}}|1\rangle\right) .
$$




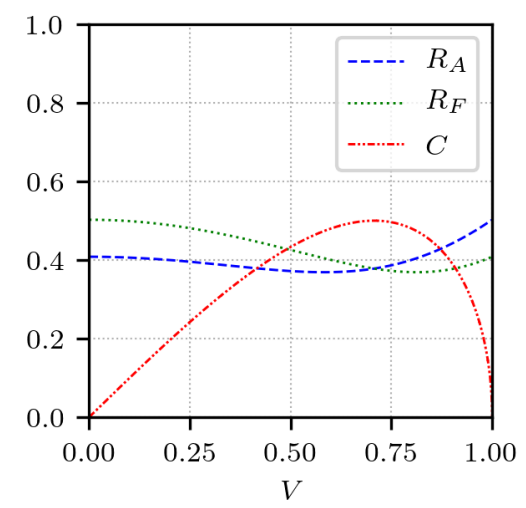

Fig. 3 Global state Concurrence, $C$, Roughness of atom, $R_{A}$, and field, $R_{F}$, as functions of the global state Visibility $V$.
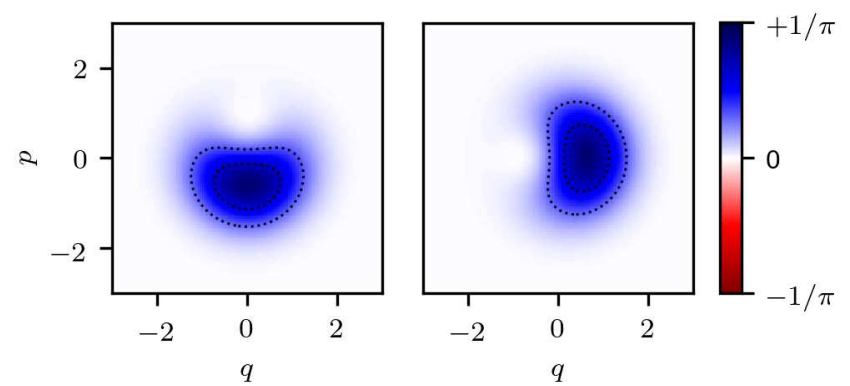

Fig. 4 The Wigner functions for the atom (left) and field (right) subsystems when $t=$ $0.5 \pi \omega^{-1}$, for the system evolved from the initial state $\left|\psi_{1}\right\rangle$ in equation (21).
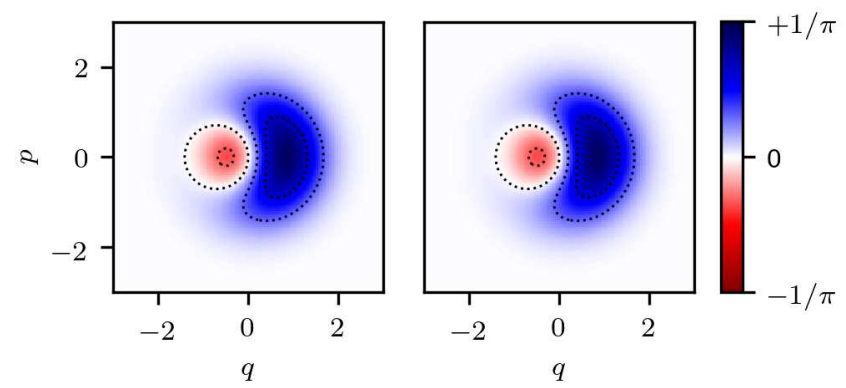

Fig. 5 The atom (left) and field (right) Wigner Functions for $\left|\psi_{2}\right\rangle$ in equation (23). 


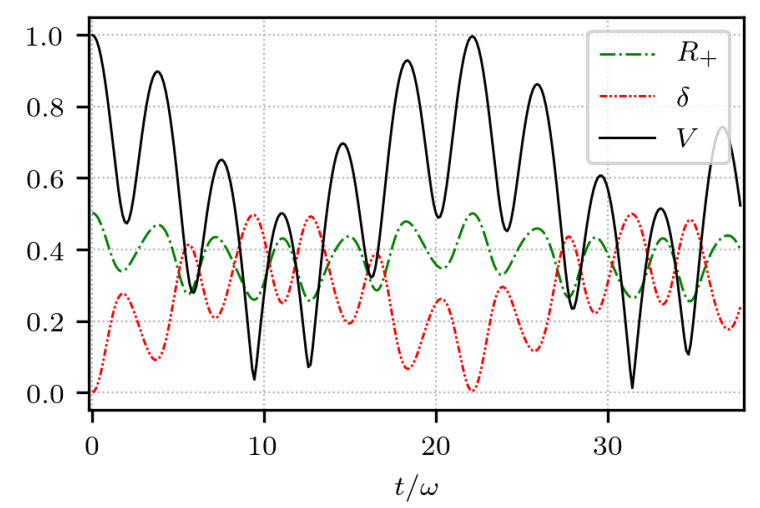

Fig. 6 Time evolution of Concurrence, $C$, combined Roughness, $R_{+}$of equation (14), and Visibility, $V$, for the initial state $\left|\psi_{2}\right\rangle$ given in equation (23).

Figure 5 gives the Wigner representation for $\left|\psi_{2}\right\rangle$ given above. Though this initial global state is that of a 2-qubits, the atom excitation will eventually be transferred to the field and in this case, one can expect for the Fock basis be populated up to $n=2$ by the dynamics. Then, a 3 by 3 density matrix must be used to represent the field degree of freedom:

$$
\rho_{F}(t)_{m, n}=B_{m}(t) B_{n}^{*}(t)+A_{m}(t) A_{n}^{*}(t),
$$

where $A_{m}$ and $B_{m}$ are given by (20). Figure 6 ilustrates the dynamic. The previous, otherwise simply periodic dynamics restricted to a 2-qubits subspace, evolve different now. The two frequencies of oscillation modulate each other and give rise to a more complex dynamics in the parameter space as can be seen in figures 7,8 and 9 .

Another initial condition of interest in this subspace is given by setting $\alpha=1, \beta=0$ and $c_{0}=c_{1}=\frac{1}{\sqrt{2}}$, which corresponds to the state:

$$
\left|\psi_{3}\right\rangle=|1\rangle \otimes\left(\frac{1}{\sqrt{2}}|0\rangle+\frac{1}{\sqrt{2}}|1\rangle\right)
$$

Figure 25 shows the Wigner representation for $\left|\psi_{3}\right\rangle$. Figure 11 shows the time evolution of the parameters. The figures 12, 13 and 14 shows the same as 7,8 and 9 but for $\left|\psi_{3}\right\rangle$. The zeroes of linear entropy shown in figure 12 corresponds to atomic pure states $\left\{|0\rangle, \frac{1}{\sqrt{2}}\left[|0\rangle+e^{i \theta}|1\rangle\right],|1\rangle\right\}$, where $\theta \in[0,2 \pi]$. The maximum of Visibility corresponds to atomic pure states $\frac{1}{\sqrt{2}}\left[|0\rangle+e^{i \theta}|1\rangle\right]$, again $\theta \in[0,2 \pi]$, with $R_{A} \approx 0.501292083$ and $\delta=0$. 


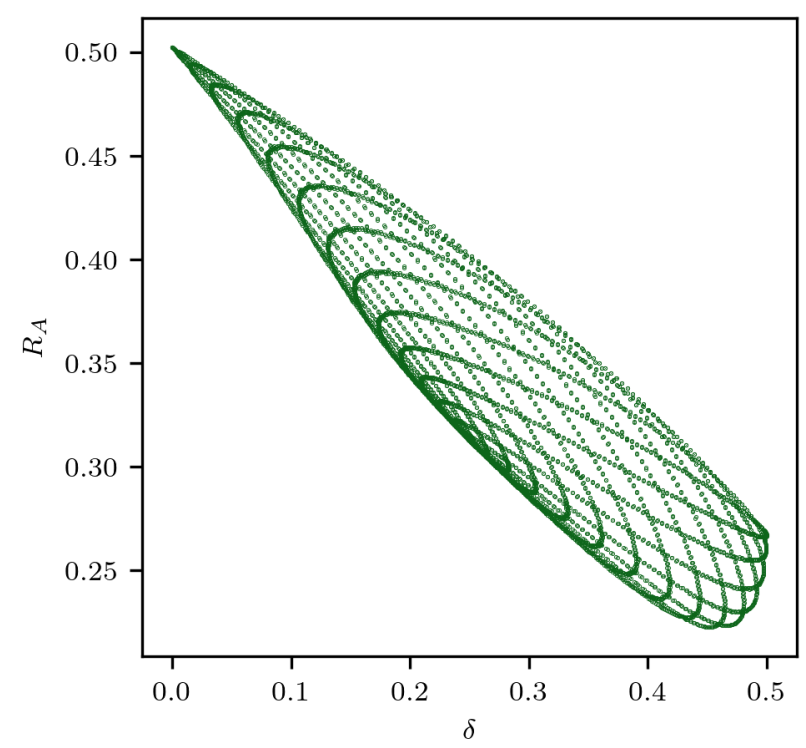

Fig. $7 \delta \times R_{A}$, evolution for the initial state $\left|\psi_{2}\right\rangle$.

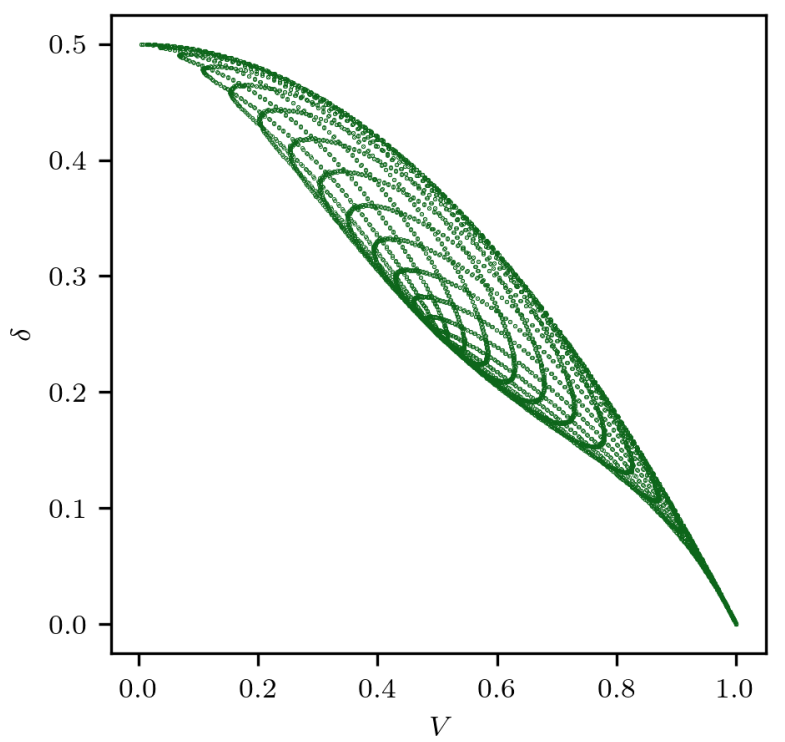

Fig. $8 V \times \delta$, evolution for the initial state $\left|\psi_{2}\right\rangle$. 


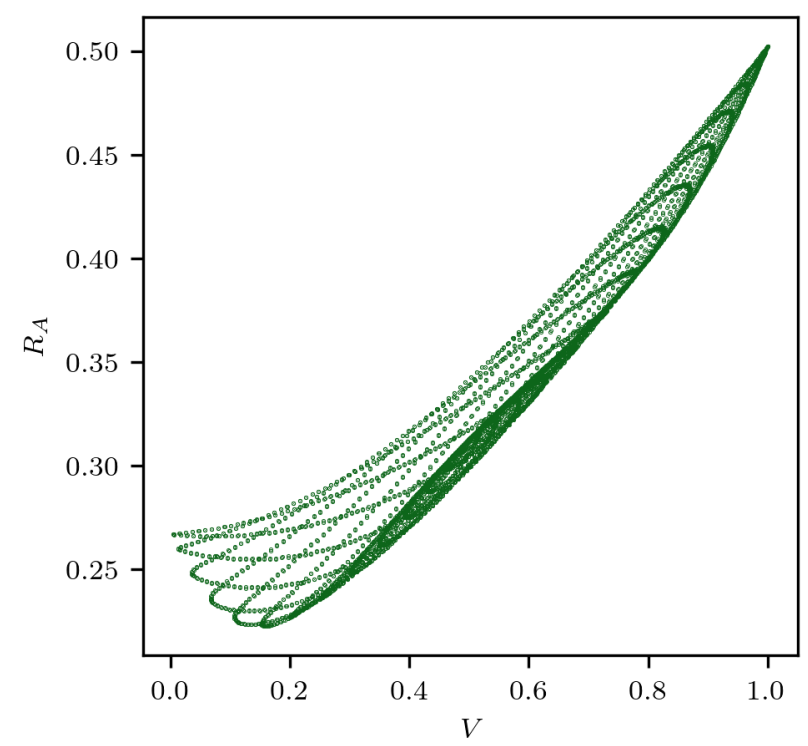

Fig. $9 V \times R_{A}$, evolution for the initial state $\left|\psi_{2}\right\rangle$.
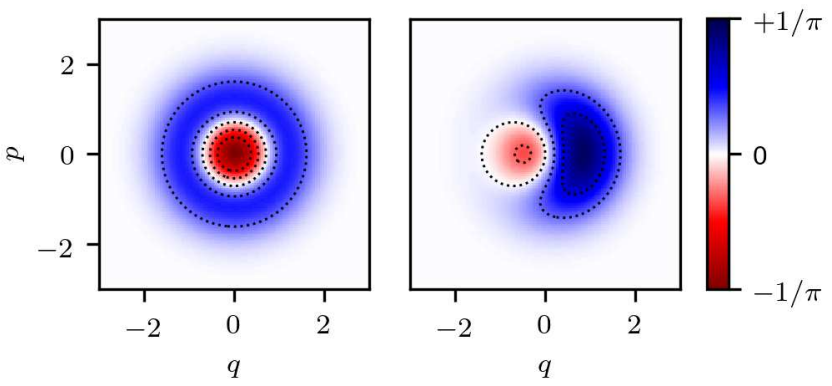

Fig. 10 The atom (left) and field (right) Wigner Functions for $\left|\psi_{3}\right\rangle$ in equation (25).

\subsection{Action of a Dispersive Environment}

Considering a phase reservoir [41] the density matrix elements are given by

$$
\begin{aligned}
& \rho_{F}(t, \Gamma)_{m, n}=\rho_{F}(t)_{m, n} e^{-\Gamma|n-m| t}, \\
& \rho_{A}(t, \Gamma)_{n, m}=\rho_{A}(t)_{m, n} e^{-\Gamma|n-m| t},
\end{aligned}
$$

where $\rho_{F}(t)_{n, m}$ is given by 24 and $\rho_{A}(t)_{n, m}$ by 19 .

The figures 15, 16, 17 and 18 shows the same as 7 and 8 but for the dispersive dynamics, with $\Gamma=5 \times 10^{-4}$ (A) and $\Gamma=5 \times 10^{-3}$ (B). As $\Gamma$ grows, the regions of high purity, roughness and high visibility are less visited. The $R \times \delta$ parameter space 16 shows that, even with the environment action, a non 


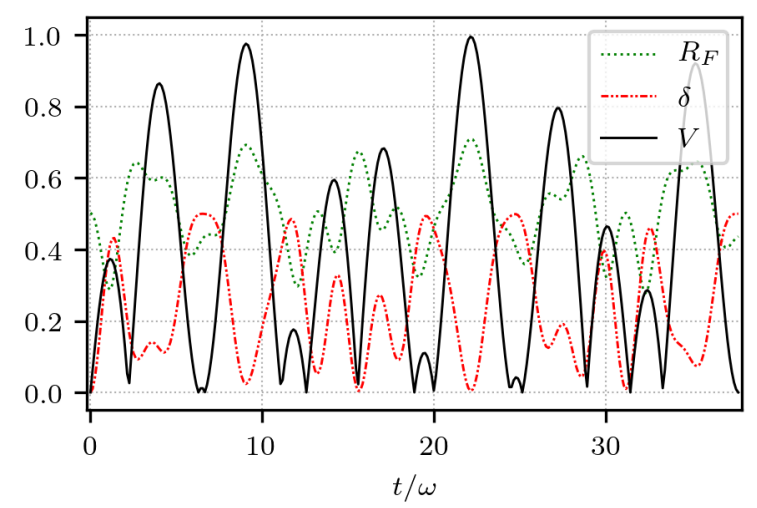

Fig. 11 Time evolution of Concurrence, $C$, combined Roughness, $R_{+}$of equation (14), and Visibility, $V$, for the initial state $\left|\psi_{3}\right\rangle$. given in equation (25).

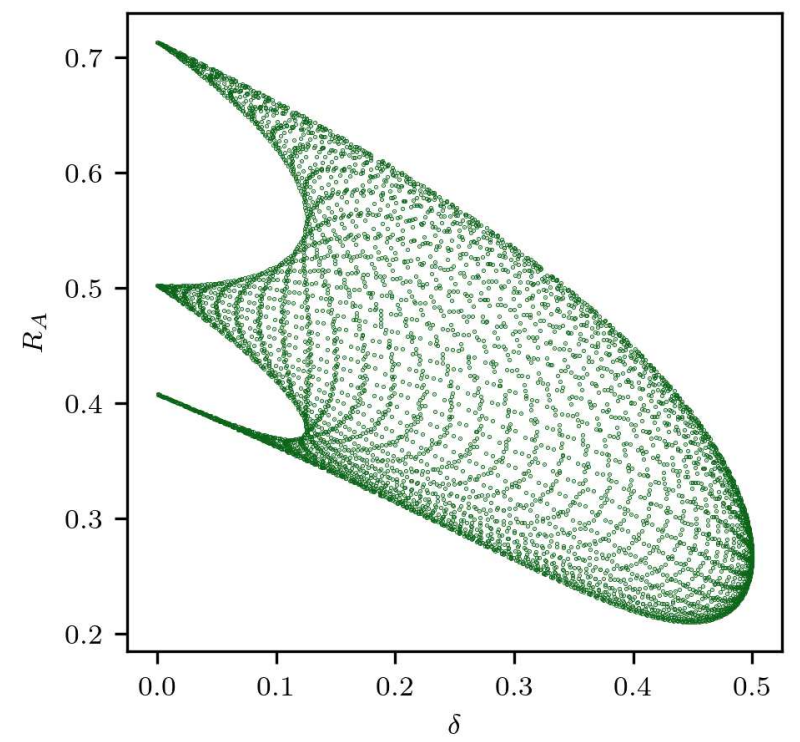

Fig. $12 \delta \times R_{A}$, evolution for the initial state $\left|\psi_{3}\right\rangle$.

pure superposition state is reached by the dynamics, it occurs when $R \approx 0.5$ and small $\delta$. As noted, there is a strong correlation between Roughness and entanglement measures, this is a consequence of the fact that Roughness is a distance based on the Wigner function and its Weierstrass transform, Husimi function, and is kown that Wigner function can be used to to visualize atomic entanglement [42]. 


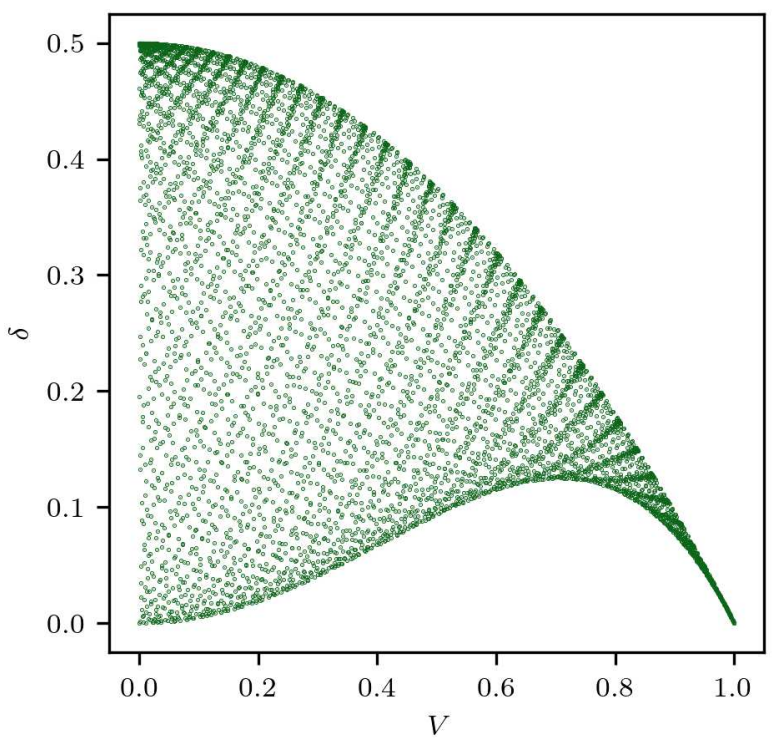

Fig. $13 V \times \delta$, evolution for the initial state $\left|\psi_{3}\right\rangle$.

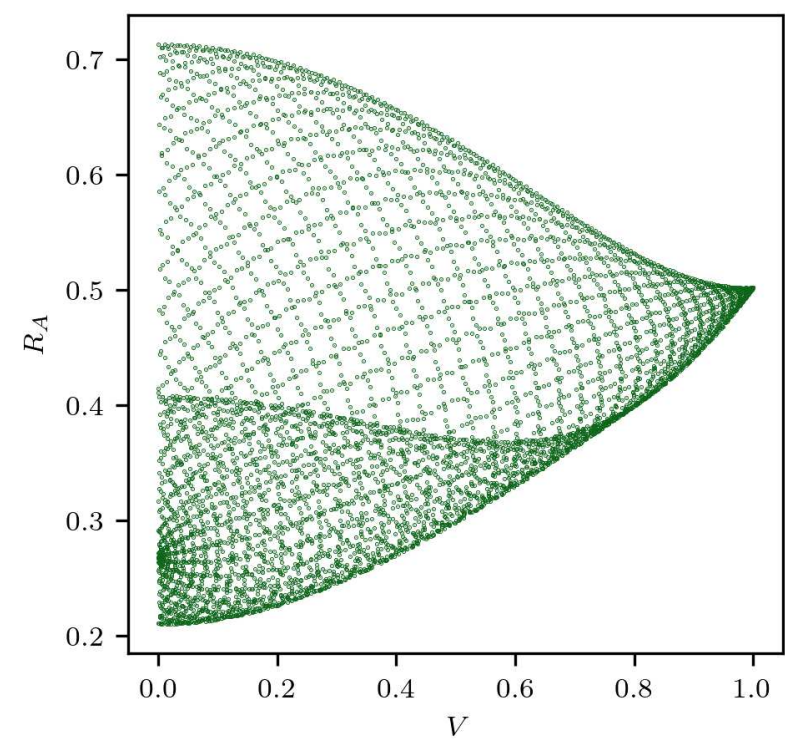

Fig. $14 V \times R_{A}$, evolution for the initial state $\left|\psi_{3}\right\rangle$. 


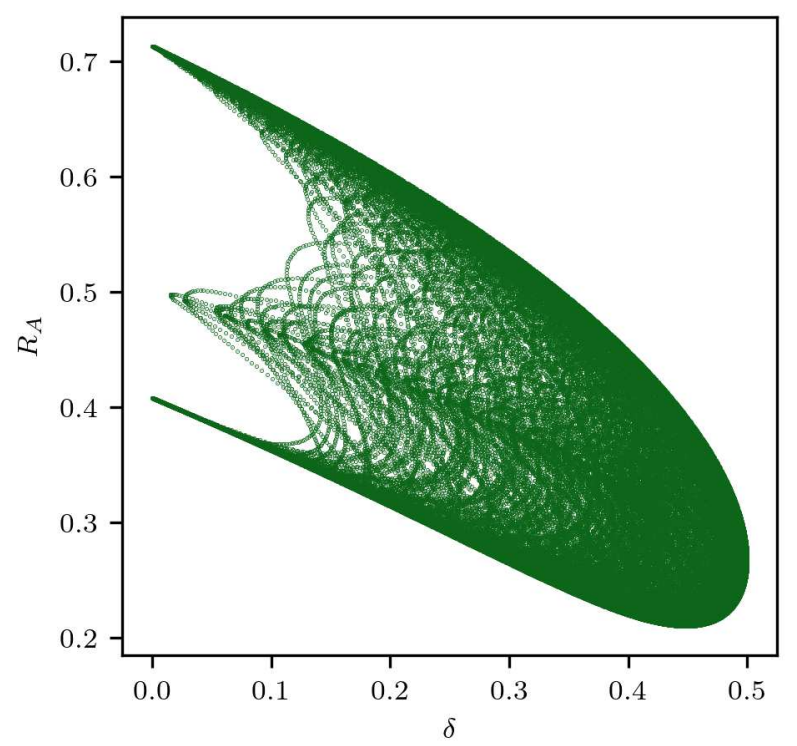

Fig. $15 \delta \times R_{A}$, evolution for the initial state $\left|\psi_{3}\right\rangle$ under a diffusive dynamics with $\Gamma=$ $5 \times 10^{-4}$.

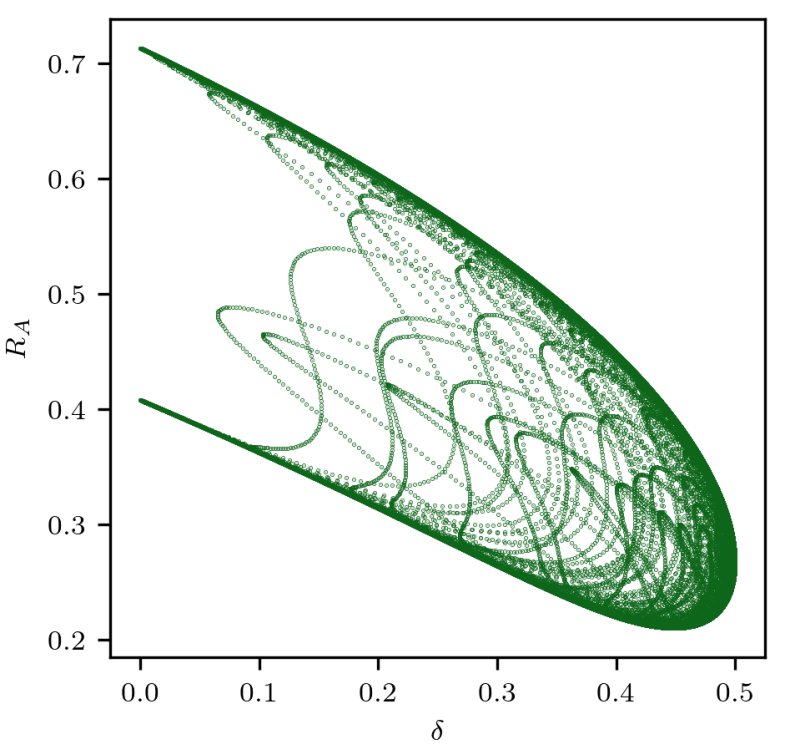

Fig. $16 \delta \times R_{A}$, evolution for the initial state $\left|\psi_{3}\right\rangle$ under a diffusive dynamics with $\Gamma=$ $5 \times 10^{-3}$.

\section{Conclusion}

As Wigner function is a state representation, it contains all information about the particle, and some information about the entanglement between particles, 


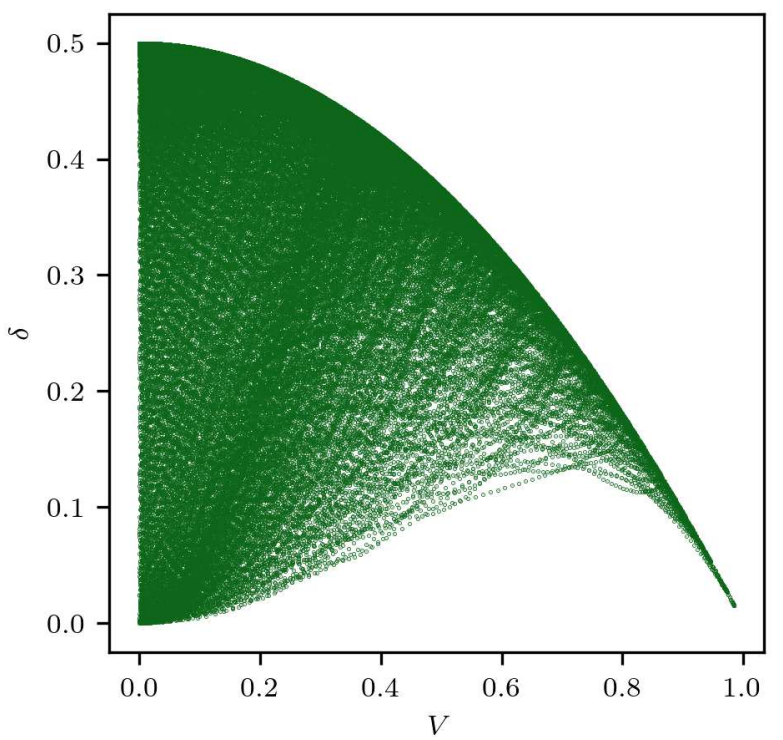

Fig. $17 \delta \times V$, evolution for the initial state $\left|\psi_{3}\right\rangle$ under a diffusive dynamics with $\Gamma=$ $5 \times 10^{-4}$.

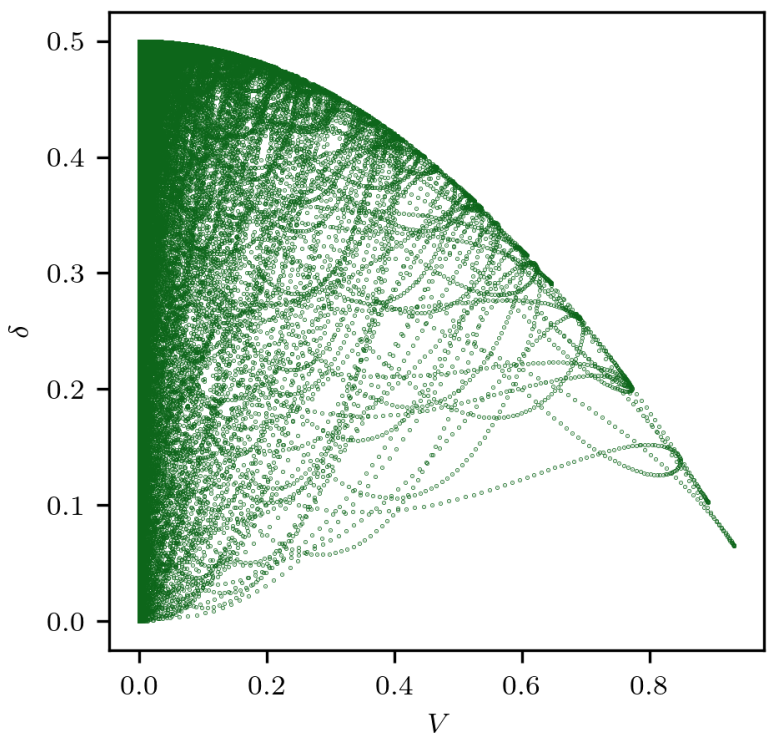

Fig. $18 \delta \times V$, evolution for the initial state $\left|\psi_{3}\right\rangle$ under a diffusive dynamics with $\Gamma=$ $5 \times 10^{-3}$

see $[43,42]$ and references there in. The Roughness is a measure that captures the main aspects of the quantum behavior, considering a distance between 
Wigner and Husimi function. The success of Roughness is due to the fact that although Husimi is a quantum distribution, it is mainly dominated by the classical dynamics since it can be approximately reproduced by classical trajectories for integrable and no-integrable dynamic [31,32]. It is important to highlight that the information about which pure states are "traversed" by the dynamics is not usually easily accessible, but in the diagram $R \times \delta$ or $R \times C$ it is clear which are the states, once they are known for their roughness. While the $\delta \times V$ diagram shows that maximum Visibility is achieved only for a pure state, from the $R \times V$ diagram we know that this state is a coherent superposition of $|0\rangle$ and $|1\rangle$. The effect of the environment destroys this superposition, although that, a non pure superposition state is obtained with low linear entropy.

\section{References}

1. A. Einstein, Dtsch. Phys. Ges. Verh. 19 (1917) 82.

2. A. Engel, A Translation of the Paper Appears, the Collected Papers of Albert Einstein, vol. 6, Trans., Princeton U. Press, Princeton, NJ, 1997, p. 434

3. Yin, J., Li, YH., Liao, SK. et al. Entanglement-based secure quantum cryptography over 1, 120 kilometres. Nature 582, 501-505 (2020). https://doi.org/10.1038/s41586-0202401-y

4. Jacak, J.E., Jacak, W.A., Donderowicz, W.A. et al. Quantum random number generators with entanglement for public randomness testing. Sci Rep 10, 164 (2020). https://doi.org/10.1038/s41567-019-0727-x

5. Arute, F., Arya, K., Babbush, R. et al. Quantum supremacy using a programmable superconducting processor. Nature 574, 505-510 (2019). https://doi.org/10.1038/s41586019-1666-5

6. Llewellyn, D., Ding, Y., Faruque, I.I. et al. Chip-to-chip quantum teleportation and multi-photon entanglement in silicon. Nat. Phys. 16, 148-153 (2020). https://doi.org/10.1038/s41567-019-0727

7. F. G. S. L. Brandão and G. Gour, Reversible Framework for Quantum Resource Theories, Phys. Rev. Lett. 115, 070503 (2015); Phys. Rev. Lett. 115, 199901 (2015).

8. Gerardo Adesso, Thomas R. Bromley, Marco Cianciaruso, Measures and applications of quantum correlations, J. Phys. A: Math. Theor. 49 473001, (2016).

9. Einstein, A.; Podolsky, B.; Rosen, N., Can Quantum-Mechanical Description of Physical Reality be Considered Complete?, Physical Review. 47 (10): 777-780, (1935).

10. Bell, JS (1982). "On the impossible pilot wave" (PDF). Foundations of Physics. 12 (10): 989-99. Reprinted in Speakable and Unspeakable in Quantum Mechanics: Collected Papers on Quantum Philosophy. Cambridge University Press, p. 160 (2004).

11. A. Omran et al. Generation and manipulation of Schrödinger cat states in Rydberg atom arrays, Science, Vol. 365, Issue 6453, pp. 570-574 (2019).

12. Gao, WB., Lu, CY., Yao, XC. et al. Experimental demonstration of a hyper-entangled ten-qubit Schrödinger cat state. Nature Phys 6, 331-335 (2010).

13. Leibfried, D., Knill, E., Seidelin, S. et al. Creation of a six-atom "Schrödinger cat" state. Nature 438, 639-642 (2005).

14. W. H. Zurek and J. P. Paz, Decoherence, chaos, and the second law. Phys. Rev. Lett. 72,2508 (1994).

15. Oliveira, A. C. and Nemes,M.C. and Romero,K.M.Fonseca, Phys Rev. E 68, 036214 (2003).

16. A. C. Oliveira and J. G. Peixoto de Faria and M. C. Nemes, Phys Rev. E 73, 046207 (2006).

17. Wootters, William K., Entanglement of Formation of an Arbitrary State of Two Qubits, Phys.Rev.Lett. 80, 2245-2248 (1998). 
18. Pranaw Rungta, V. Buz?ek, Carlton M. Caves, M. Hillery, and G. J. Milburn, Universal state inversion and concurrence in arbitrary dimensions, Phys Rev. A 64, 042315 (2001).

19. A.R. Bosco de Magalhães, Adélcio C. Oliveira,Characteristic entanglement timescales of a qubit coupled to a quartic oscillator, Physics Letters A, V. 380, 4,Pages 554-561, (2016).

20. A. R, Bosco de Magalhães and A. C Oliveira, Phys. Scr. 86 035001, (2012).

21. M.C. Nemes, K. Furuya, G.Q. Pellegrino, A.C. Oliveira, M. Reis, L. Sanz, Quantum entanglement and fixed point Hopf bifurcation,Physics Letters A, V; 354, Pages 60-66, (2006).

22. G. Manfredi and M. R. Feix Phys. Rev. E 62, 4665, (2000).

23. A. Kenfack, K. Zyczkowski, Negativity of the Wigner function as an indicator of nonclassicality, J. Opt. B, Quantum Semiclass. Opt. 6(10), 396 (2004).

24. H. Lemos, A. Almeida, B. Amaral and A. Oliveira, "Roughness as classicality indicator of a quantum state," Physics Letters A, vol. 382, no. 12, pp. 823-836, (2018).

25. Luo, Shunlong and Zhang, Yue, Quantifying nonclassicality via Wigner-Yanase skew information,Phys. Rev. A, v. 100, 032116, (2019).

26. E.T. Jaynes; F.W. Cummings, "Comparison of quantum and semiclassical radiation theories with application to the beam maser". Proc. IEEE. 51 (1) (1963).

27. J. Gea-Banacloche, Collapse and revival of the state vector in the Jaynes-Cummings model: An example of state preparation by a quantum apparatus, Phys. Rev. Lett. 65, 3385, 1990.

28. J. Gea-Banacloche, Phys. Rev. A 44, 5913 (1991).

29. M. Jakob, J. A. Bergou, Quantitative complementarity relations in bipartite systems: Entanglement as a physical reality, Optics Communications, Volume 283, Issue 5,pp. 827-830 (2010).

30. N. Schwaller, M. Dupertuis, and C. Javerzac-Galy, Evidence of the entanglement constraint on wave-particle duality using the IBM Q quantum computer, Phys. Rev. A 103, 022409 (2021).

31. Classical Structures in the Husimi Distributions of Stationary States for $\mathrm{H}_{2}$ and $\mathrm{HCl}$ Molecules in the Morse Potential. A. C Oliveira and M C Nemes 2001 Phys. Scr. 64279

32. A.C. Oliveira, Semiclassical Husimi function of simple and chaotic systems, Journal of Modern Physics 3 (8), 694-701

33. A. Wunsche, V. V. Dodonov; O. V. Man'ko, and V. I. Man'ko, Fortschr. Phys. 49 (2001) 10-11, 1117-1122

34. Leslie E. Ballentine. The statistical interpretation of quantum mechanics. Rev, Mod. Phys., 42(4):358, 1970.

35. Leslie E. Ballentine, Yumin Yang, and J. P. Zibin. Inadequacy of Ehrenfest's theorem to characterize the classical regime. Phys. Rev. A, 50(4):2854, 1994.

36. L. E. Ballentine and S. M. McRae. Moment equations for probability distributions in classical and quantum mechanics. Phys. Rev. A, 58(3):1799, 1998.

37. L. E. Ballentine. Lyapunov exponents for the differences between quantum and classical dynamics. Phys. Rev. A, 63(2):024101, 2001.

38. Nathan Wiebe and L. E. Ballentine. Quantum mechanics of Hyperion. Phys. Rev. A, $72(2): 022109,2005$

39. M. Reis and A. C. Oliveira, "Roughness as Entanglement Witness: The two Coupled Cavity Model," 2018 SBFoton International Optics and Photonics Conference (SBFoton IOPC), Campinas, Brazil, pp. 1-5, (2018).

40. J. M. Raimond, M. Brune, and S. Haroche, Rev. Mod. Phys. 73, 565 (2001).

41. A. C. Oliveira and A. R. Bosco de Magalhães Phys. Rev. E 80, 026204 (2009).

42. B. I. Davies et al, Visualizing spin degrees of freedom in atoms and molecules Phys. Rev. A 100, 042102

43. J. Weinbub and D. K. Ferry, Recent advances in Wigner function approaches, Appl. Phys. Rev. 5, 041104 (2018) 


\section{Declarations}

6.1 Funding

The authors gratefully acknowledge the support of the Brazilian Agency Fundação de Amparo à Pesquisa do Estado de Minas Gerais (FAPEMIG) through grant No. APQ-01366-16.

6.2 Conflicts of interest/Competing interests

There is no conflict of interest.

6.3 Availability of data and material

Not applicable

6.4 Code availability (software application or custom code)

It was used an open-source code QUTIP

6.5 Authors' contributions

The work was equally divided by the authors. 